\title{
Introduction to Special Issue ASD in Adulthood: Comorbidity and Intervention
}

\author{
Susan W. White ${ }^{1} \cdot$ Antoinette Sabatino DiCriscio $^{2}$
}

Published online: 30 October 2015

(c) Springer Science+Business Media New York 2015

The intent of this special issue was to shine a spotlight on research examining the assessment and treatment of cooccurring problems among adults with ASD. As the number of adults with ASD has risen, the need for researchbased guidance on how to best meet this population's needs has become apparent. This growing need is evident in the rate of scholarly productivity in this area. A cursory GoogleScholar search reflects the upsurge in scientific interest in this area. A search of 'adult autism' yields 21,000 results from 2014 to October 1, 2015. This doubles what was seen just a decade earlier (2004-2005), for which a search with the same search terms yields only 10,900 results. In the summer of 2014 , we issued a call for submissions for this special issue on ASD in Adulthood, with a focus on Comorbidity and Intervention. We had many excellent submissions, and all were subjected to $J A D D$ 's standard masked peer review process.

The ten articles comprising this special issue fall into four broad domains, which can serve as a blueprint for future research on some of the key issues our field faces. The first domain broadly relates to diagnostic evaluation of ASD in adults and diagnostic stability. Louwerse and colleagues examined the stability of ASD dimensionally,

Susan W. White

sww@vt.edu

Antoinette Sabatino DiCriscio

asdicriscio@geisinger.edu

1 Department of Psychology, Child Study Center, Virginia Tech Center for Autism Research, 460 Turner St., Suite 206, Blacksburg, VA 24060, USA

2 Autism and Developmental Medicine Institute, Geisinger Health System, 120 Hamm Drive, Suite 2A, Lewisburg, PA 17837, USA using the calibrated severity scores of the ADOS. They found that stability over a 7-year period was fairly high, from childhood (mean age 9 years) into adolescence (mean age 16 years). Consistent with prior research in this area, they found that although ASD is diagnostically stable, dimensionally there are fluctuations in severity of ASD over the life course. Additionally, most of the individuals who no longer met diagnostic criteria for ASD at the 7-year follow-up had other psychiatric disorders. Louwerse and colleagues did not, however, find evidence for a linear relationship between ASD severity and psychiatric comorbidity. The mechanistic relationship between ASD severity and the emergence or presence of other disorders is complex, and further work is needed at both the diagnostic and dimensional levels to better understand how ASD, and secondary symptoms, change developmentally. Using a large, well-characterized multi-site sample, Pugliese and colleagues conducted a replication of the Autism Diagnostic Observation Schedule (ADOS-2, Lord et al. 2012) revised diagnostic algorithm (Hus and Lord 2014). They found that the 2014 revised algorithm yielded increased sensitivity but lower specificity. Interesting group patterns in their sample emerged. For example, specificity was lowest for adults of average cognitive ability, and both sensitivity and specificity were higher among females relative to males. The authors concluded that best practice with adults, similar to that for children (Dunn 2000; Le Couteur et al. 2008), is use of the ADOS in conjunction with other methods of evaluation, to support accurate differential diagnosis.

In the second domain, we have five articles pertaining to psychiatric comorbidity, including its expression, assessment, and relationship to core ASD symptom severity. Kreiser and White, in an analogue sample of healthy adults, found that ASD traits positively predicted likelihood of 
exceeding diagnostic threshold for mood disorder, consistent with extant research indicating high rates of depression among individuals with ASD (e.g., Gotham et al. 2015a). This prediction was moderated by gender, such that women with high levels of ASD traits were more likely to meet criteria for depression. The specificity of the interaction for mood disorder (and not for other types of disorder) suggests there may be something unique about female risk for mood disorder in the context of ASD. Greaves-Lord and colleagues, in their longitudinal study of children diagnosed with ASD, found that comorbidity was stable within domain such that externalizing problems during childhood did not develop or transition into internalizing problems from childhood to adolescence. In general, comorbidity declined from childhood to adolescence. Variables related to the ASD diagnosis, specifically reduced social interest and stereotyped behavior, predicted greater stability of psychiatric problems.

Maddox and White, the third article in this domain, explored the phenomenon of social anxiety among adults with ASD. They found that $50 \%$ of non-treatment seeking adults met diagnostic criteria for SAD. Relative to adults with ASD who did not also have SAD, those with comorbid SAD had more severe ASD symptomatic impairment, as rated by the SRS, which indirectly supports a relationship between ASD—related social disability and social anxiety. On the whole, Maddox and White found there to be considerable similarity in the progression and expression of SAD between adults with ASD and those without. Cervantes and Matson, in the fourth article in this area, examined the influence of co-occurring ASD on comorbid mental health symptoms among adults with severe or profound intellectual disability. They found that presence of ASD increases vulnerability for secondary symptoms across a range of disorders. This study is critical as so much of our research on comorbidity within ASD has been constrained to individuals without co-occurring ID. Comorbidity is not confined to adults who are higher functioning and further research is needed to determine how to best assess and treat secondary symptoms in minimally verbal and cognitive impaired adults. Finally, Swain and colleagues tested a theorized model of the role of emotion dysregulation and social motivation on the experience of social anxiety. Using a treatment-seeking sample, they found that both social motivation and emotion dysregulation predicted social anxiety, but they found no evidence for an interaction, or moderating effects of social motivation. Taken together, these articles highlight the importance of continuous evaluation of comorbid psychiatric conditions in ASD-especially as individuals progress through adolescence and into early adulthood, when transitions and novel contexts could give rise to or reveal cooccurring maladaptive traits.
The two articles in the third domain concern intervention and service delivery, a sorely under-researched area in adulthood ASD (e.g., Howlin and Taylor 2015). Laugeson and colleagues reported outcomes from a randomized controlled trial of the social intervention program, PEERS for Young Adults (Laugeson 2014). Significant improvement on multiple indices of social functioning were reported as well as evidence for sustained effects 16 weeks after treatment completion. These findings, though preliminary, are crucial to our growing understanding of the malleability of the core social disability domain in ASD. Schall and colleagues compared adults who had completed an organized program (Project SEARCH) to those in supported employment settings who had not received any programming. The results of this novel study are illuminating: adults with ASD have the ability to secure and maintain paid employment when provided with intensive and personalized support. The authors offered suggestions for future research on promoting successful employment outcomes, such as intensive internship experiences that help prepare adults for the challenges and novelty of firsttime employment, such as completing assigned tasks and managing potential interruptions or suspending interactions with others until tasks have been successfully completed.

In the final article, Zerbo and colleagues present findings of a study that directly speaks to the need for clearer, action-oriented public policy and improved training of providers. They found that physicians treating adults with ASD report having insufficient training and skills on how to best care for adults with ASD. One of the biggest areas of concern for providers is in how to effectively communicate with their adult patients with ASD.

The research described herein is exciting as well as formative to our evolving understanding of the psychiatric complexity of adults with ASD. Collectively, the findings of these studies build upon a body of research demonstrating that outcomes in multiple domains (e.g., vocational, educational), functional independence, and quality of life are severely hampered for adults with ASD (Gotham et al. 2015b; Howlin 2003; Lawrence et al. 2010). Risk for adverse outcomes is likely increased in the context of psychiatric comorbidity. Although there is clearly much more work to be done, we appreciate the fluidity in ASD symptoms over the lifecourse (despite stability in diagnosis), the importance of assessing for comorbid conditions (although we lack sensitive tools with which to assess comorbidity), and the need for more research on treatment of core and secondary symptoms, and training of direct care providers.

Author Contributions SWW and AS jointly developed the special issue, served as guest editors, and collaborated in the writing of this Introduction. Neither SWW or AE served as guest editor for any submission on which they were a co-author. 


\section{References}

Dunn, G. (2000). Statistics in psychiatry. London: Arnold.

Gotham, K., Brunwasser, S. M., \& Lord, C. (2015a). Depressive and anxiety symptom trajectories from school age through young adulthood in samples with autism spectrum disorder and developmental delay. Journal of the American Academy of Child and Adolescent Psychiatry, 54(5), 369-376. doi:10.1016/j. jaac.2015.02.005.

Gotham, K., Marvin, A. R., Taylor, J. L., Warren, Z., Anderson, C. M., Law, P. A., et al. (2015b). Characterizing the daily life, needs, and priorities of adults with autism spectrum disorder from Interactive Autism Network data. Autism. doi:10.1177/ 1362361315583818.

Howlin, P. (2003). Outcome in high-functioning adults with autism with and without early language delays: Implications for the differentiation between autism and Asperger syndrome. Journal of Autism and Developmental Disorders, 33, 3-13. doi:10.1023/ A:1022270118899.

Howlin, P., \& Taylor, J. L. (2015). Addressing the need for high quality research on autism in adulthood. Autism, 19(7), 771-773. doi:10.1177/1362361315595582.
Hus, V., \& Lord, C. (2014). The autism diagnostic observation schedule, module 4: Revised algorithm and standardized severity scores. Journal of Autism and Developmental Disorders, 44(8), 1996-2012.

Laugeson, E. A. (2014). The PEERS ${ }^{\circledR}$ curriculum for school based professionals: Social skills training for adolescents with autism spectrum disorder. New York, NY: Routledge.

Lawrence, D. H., Alleckson, D. A., \& Bjorklund, P. (2010). Beyond the roadblocks: Transitioning to adulthood with Asperger's disorder. Archives of Psychiatric Nursing, 24(4), 227-238.

Le Couteur, A., Haden, G., Hammal, D., \& McConachie, H. (2008). Diagnosing autism spectrum disorders in pre-school children using two standardised assessment instruments: the ADI-R and the ADOS. Journal of Autism and Developmental Disorders, 38(2), 362-372.

Lord, C., Rutter, M., DiLavore, P. C., Risi, S., Gotham, K., \& Bishop, S. (2012). Autism diagnostic observation schedule, 2nd edition (ADOS-2). Torrance: Western Psychological Services. 